\title{
Translating COVID-19 Pandemic Surge Theory to Practice in the Emergency Department: How to Expand Structure
}

\author{
Matteo Paganini, MD (10; Andrea Conti, MD (1); Eric Weinstein, MD, MScDM; \\ Francesco Della Corte, MD; Luca Ragazzoni, MD, PhD
}

\section{ABSTRACT}

Multiple professional societies, nongovernment and government agencies have studied the science of sudden onset disaster mass casualty incidents to create and promote surge response guidelines. The COVID-19 pandemic has presented the health-care system with challenges that have limited science to guide the staff, stuff, and structure surge response.

This study reviewed the available surge science literature specifically to guide an emergency department's surge structural response using a translational science approach to answer the question: How does the concept of sudden onset mass casualty incident surge capability apply to the process to expand COVID-19 pandemic surge structure response?

The available surge structural science literature was reviewed to determine the application to a pandemic response. The on-line ahead of print and print COVID-19 scientific publications, as well as gray literature were studied to learn the best available COVID-19 surge structural response science. A checklist was created to guide the emergency department team's COVID-19 surge structural response.

Key Words: COVID-19, pandemics, SARS-CoV-2, surge capacity, translational science

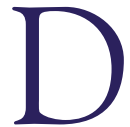
uring the 20th century, health care has radically evolved due to new discoveries and changes in global politics, while the percentage of population that gained access to health care has dramatically increased. Despite this achievement, governments strain to fulfill the overwhelming request for medical assistance. Each government deploys resources trying to meet United Nations' Sustainable Development Goal No. 3 (Ensure healthy lives and promote well-being for all at all ages) ${ }^{1}$ while balancing health-care expenditure within their gross domestic product. ${ }^{2}$ Health-care systems oscillate between 2 key elements: demand and available resources. Similar to living organisms, health care has complex mechanisms to achieve and maintain a "stability of the internal environment" (milieu intérieur), despite external stressors. ${ }^{3}$ The resulting equilibrium could resemble cellular homeostasis during normal conditions. ${ }^{4}$ However, extraordinary events can result in a significant imbalance.

The novel coronavirus (SARS-CoV-2) pandemic is currently threatening several national health systems. ${ }^{5}$ To date, Italy has been one of the most affected countries ${ }^{6}$ where public health departments, emergency medical systems, and hospitals are struggling to deal with the surge of patients affected by $2019-\mathrm{nCoV}$.
Unfortunately, contagion rates are estimated to rise exponentially in many countries, regardless of their health-care delivery system. Urgent actions are required to modify both health-care systems configurations and hospitals' capacity and capability to respond. In such situations, high-quality data are limited. The application of translational science in this disaster medicine setting can provide stakeholders and clinicians with acceptable evidence-based medicine concepts. $^{7-9}$

- $\mathrm{TO}=$ Identification of opportunities and approaches to a health problem. How does the concept of sudden onset mass casualty incident (MCI) surge capability apply to the process to expand COVID-19 pandemic surge structure response? Structure refers to the physical location of the space where providers attend to the patient.

- $\mathrm{T} 1=$ Basic research for clinical effect and/or applicability, human physiology knowledge, and potential for intervention. Review prior MCI surge literature for specific references to surge structure reconfiguration guidance.

- $\mathrm{T} 2=$ New interventions to form basis for clinical application and evidence-based guidelines. Scoping Literature Review of available COVID-19 peer review and gray literature. ${ }^{10}$ 
FIGURE

\section{Pandemic Surge Timeline.}

\begin{tabular}{|c|c|c|}
\hline & Structure & Space \\
\hline Conventional & Same & (1) \\
\hline Contingency & Same & (1) 1 \\
\hline Crisis & Additional & (1) 1 \\
\hline
\end{tabular}

Structure Emergency Department

Space Room or area to attend to a patient or patients

(-) During a pandemic the exponential growth magnitude over time in response to the demand of increasing patient volume

- T3 = Implementation of research findings in clinical practice. Creation of a checklist for COVID-19 Surge Structure Planners.

- $\mathrm{T} 4=$ Effects on practice influencing populations and policy. Collection of completed checklists to improve the COVID-19 Surge Structure Planning.

\section{THE TO QUESTION}

A marked increase in demand for medical resources, known as "surge,"11 can have detrimental effects on health-care systems if the supply of available resources to meet this demand, known as surge capacity, is not sufficient. ${ }^{12}$ Surge capability is how surge capacity is used to meet the unexpected surge. ${ }^{11} \mathrm{With}$ this perspective, the concept of resilience is more appropriate to represent the properties of health-care systems during disasters or MCIs. Initially, this engineering concept described the elastic deformation of materials under physical strain. ${ }^{13}$ This term was implemented in sociology as the ability of groups or communities to cope with external stresses and disturbances. ${ }^{14}$ Resilience can be applied to public health to illustrate the adaptations at individual, community, and system levels. ${ }^{15}$ A resilient health-care system must be able to limit and cope with stressors and events (absorptive capacity), to adapt itself toward external events (adaptive capacity), to forecast events and take action to minimize effects (anticipatory capacity), and to change the structures and operations to better address results (transformative capacity). ${ }^{15}$ Altogether, the improvement of surge capability and resilience are critical steps of disaster mitigation and preparedness, to achieve an adequate response toward sudden and high-impact events, such as disasters or outbreaks. ${ }^{16}$

\section{THE T1 REVIEW}

\section{Mass Casualty Incidents}

After the Oklahoma City Bombing in 1995, ${ }^{17}$ there was renewed interest in MCI management, specifically to create plans to meet the demand of a sudden supply of patients after a sudden onset terrorist disaster. Emergency Departments (EDs) would have to be able to rapidly expand the operational staff, stuff, and structures. ${ }^{18}$ This study led to the terrorism $\mathrm{MCI}$ response to factor the resilience of the first responders and the first receivers to not become terrorist victims in addition to being able to treat the overwhelming demand of injured patients. The staff, stuff and structures would have to protect all concerned.

The MCI planning and response to chemical (Aum Shinrikyo ${ }^{19}$ ) and biologic $\left(\right.$ Anthrax ${ }^{20}$ ) terrorism added to the mitigation, preparation, and response calculus. Materials to protect staff, personal protective equipment, along with training and competencies, would have to be funded and maintained for all potentially affected staff. The Severe Acute Respiratory Syndrome (SARS) epidemic of 2002 $2004^{21}$ moved the MCI discussion from sudden onset disaster (SOD) planning and response to the unique variables to meet the demand of a sudden onset of patients with a novel disease, or a disease caused by an agent that was not known and had no specific diagnostic test or treatment. This response entailed a new calculus to prevent transmission to Emergency Medical Services (EMS) staff or emergency department (ED) and hospital patients, visitors, and staff. The 2009 H1N1/09, ${ }^{22}$ Middle East Respiratory Syndrome (MERS) in $2012,{ }^{23}$ and Ebola in $2013^{24}$ tested the mettle of governments, first responders, and receivers, as well as those health-care systems.

\section{Pandemic Surge Capacity}

In 2009, planners began to adapt the structural phases in the timeline $^{25}$ of a sudden onset MCI. Adapting this to the timeline of a COVID-19, with days to weeks before the overwhelming demand of patients present with hypoxia and respiratory failure (Figure 1).

\section{Conventional}

When the ED is operating with typical volume or even if the ED is boarding patients, the staff can divert patients that fit the COVID-19 "person under investigation" from the entrance to the ED, bypassing triage into a room with a door or an Airborne Infection Isolation Room (AIIR) if available with standard precautions. ${ }^{26}$ During the time this patient or a few more present with similar symptoms and are evaluated, other typical ED patients are also arriving. The ED staff resilience features: strict efforts to not expose any ED patients and staff while creating ED space for these patients through an expedited discharge process of all other patients to designated 
ED areas waiting for rides; hospital admission process, including abbreviated reports or orders to the in-patients units; and other creative efforts, such as moving patients to the hallways that typically are not used when the ED is overcrowded. The ED structure adapts to meet the demand with present ED patients continuing to be processed accordingly, albeit delayed, without compromising care.

\section{Contingency}

Sudden onset disasters typically have a finite number of patients involved, although the treating first responders and first receivers are not aware of this as the $\mathrm{MCI}$ evolves. When this demand exceeds the daily supply of staff, stuff, and structures due to sheer volume and presenting pace of patients layered on the usual daily census, the conventional $\mathrm{MCI}$ response expands. Circumstances such as patient acuity or particular incidents such as burn, blast, or chemical injuries, as well as accompanying familiar blunt or penetrating trauma, now has exceeded the configured space within the structure of the ED. ED staff resilience will seek other structures with space to accommodate the staff and stuff to attend patients within the hospital, approximate to the ED or in the space outside the ED itself. This may have been planned and exercised, or discussed during exercises, to be adapted to meet the demand. If the MCI has not been encountered, exercised, or planned, then ED leadership will discover and adapt as the MCI unfolds: nearby hallways, a family conference room, ED offices that are empty or easily emptied to accept the staff and stuff to attend to an $\mathrm{MCI}$ patient. Current ED rooms are repurposed or patients are cohorted, ${ }^{27}$ those pending test results or rides home are placed in nontreatment areas; ED staff coordinates to adapt other treatment structures like the preoperative and postoperative units, or intensive care unit (ICU) level of care rooms. While the MCI efforts are within the ED or have expanded, the current ED and newly arriving ED patients continue to occupy their initial structure, although space may have contracted to continue to deliver functionally equivalent medical care, with minimal increase in risk to the patient. ${ }^{28}$

These strategies are used for the continued rise in the number of patients presenting with COVID-19 symptoms that requires the strict measure to limit exposure. Rooms with doors are a must, personal protective equipment must be available, and the flow of staff has to be managed to limit exposure.

\section{Crisis}

Sudden onset disasters have a finite number of patients concentrated in an initial peak, with the supply of staff and stuff that arrive over time to meet the demand. Most patients can be treated and discharged, while those admitted can be cohorted into space that suits their needs without the strict requirement of standard precautions, unlike the COVID-19 response that will require proper standard precautions. The overall
MCI visits will decline over time, MCI hospital admissions will decrease, and the ED volume will eventually resume usual operation levels. This is in distinction to a viral outbreak that will have a gradual onset of those seeking medical attention. While facing an incoming epidemic or pandemic, hospitals can adapt progressively as long as the contagion rates are predictable through estimates that guide stakeholders in the process. ${ }^{29}$

An earthquake in a major metropolitan area could create a catastrophic scenario that would require resources that would have to come from outside the region due to the sheer number of patients. As the COVID-19 pandemic has progressed in each city, the sheer number of patients arriving has been described as "like an earthquake every day" 30 with an unpredictable increase while there is a lack of inpatient discharges. The resilience of the ED will require a formal process to create the structures with the space to accommodate the COVID-19 oxygen requiring patients, noninvasive ventilators, or ventilators that are accumulating due to a lack of in-patient space.

\section{T2: SCOPING LITERATURE REVIEW OF CURRENT COVID-19 LITERATURE}

The publication of COVID-19 peer-reviewed articles and gray sources is ongoing. This information will demonstrate how hospitals are expanding their surge structure. A literature review was conducted according to the Preferred Reporting Items for Systematic Reviews and Meta-Analyses extension for Scoping Reviews (PRISMA-ScR) checklist, ${ }^{31}$ to include manuscripts published up to 22 March 2020; and if not in print then accepted for online publication ahead of print.

\section{Search Strategy}

The controlled vocabulary of Medical Subject Headings (MeSH) from PubMed, including subheadings, publication types, and supplementary concepts, was used to identify the entry terms for the search (Figure 2). ${ }^{32}$

The search was performed on PubMed/MEDLINE, Scopus, and Google Scholar from the 15 to 22 March 2020. A search of the gray literature was conducted at the same time.

Table 1 and Figure 3 were developed to extract data based on the above review of prior $\mathrm{MCI}$ and pandemic surge capacity as discussed earlier.

Inclusion criteria were as follows: (1) studies describing hospital reaction to the SARS-CoV-2 pandemic and specifically detailing structural changes and infrastructural remodeling to cope with the new challenges imposed by the outbreak; (2) any study design, reports included; and (3) gray literature, also including professional society guidelines, protocols, or consensus statements, peer-reviewed blog posts, and podcasts. 
FIGURE 2

Search Query.
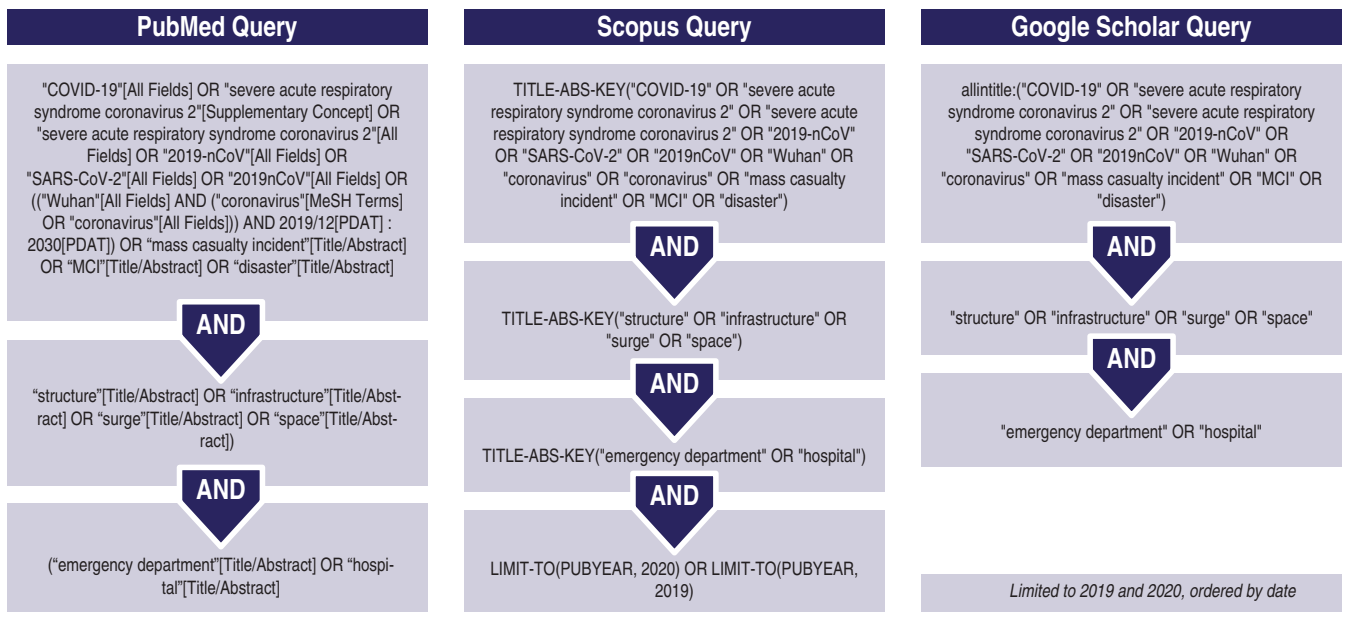

Exclusion criteria were as follows: (1) contents in languages other than English, French, or Italian; and (2) literature without available abstract or full-text.

\section{Search Findings}

Our search did not identify manuscripts to be included in the qualitative analysis. The records identified through gray literature scanning ${ }^{30,33-41}$ are summarized in Table 2.

The retrieved materials were mostly found on websites of national and international professional societies. Of note, 2 podcasts ${ }^{30,33}$ and 1 blog post ${ }^{34}$ were found reporting structural adaptations of the ED of the hospital of Bergamo, Italy.

Sources wrote of the need to create dedicated pathways to divide potentially infectious patients (COVID-19) the others (Clean) presenting to the ED. Among the other suggestions made, the management of spaces should take into account the increased prevalence of the disease, thus requiring a track dedicated to respiratory symptoms (reported as approximately $90 \%$ of current ED activity in Bergamo hospital). ${ }^{30}$ Physical barriers should actively separate the 2 fluxes of patients.

The 2 articles are pending online publication ahead of print in the Disaster Medicine and Public Health Preparedness journal (personal communication). Medicine relies on tradition, and this is evident in the observation of Faccincani ${ }^{42}$ of his experience in Milan. Treatment of hemorrhagic shock calls for transfusion of " 2 units of packed red blood cells and tell the blood bank to stay 2 units ahead" theory applies to his experience to stay 1 ventilator ahead. Extending this to the awareness of when to start seeking more structure for treatment space is when you are comfortable to be aware of the period of time that would require a structure to be adapted, repurposed,

or created. The number of references that assign number of patients to be prepared to treat during the timeline of surge, from conventional to contingency to crisis, in peer-reviewed literature, professional societies, nongovernment and government publications is far too great to cite. Only a hospital that is studying their trends can determine when to begin the reconfiguration of a structure. The caution to stay "1 structure ahead" seems to be prudent. When the team has identified structures within their facility to reconfigure, the time that this would take is part of the calculus when prioritizing when to expand the ED COVID-19 footprint.

Gagliano et al. ${ }^{43}$ wrote of their experience in the Northern City of Lodi, the epicenter of the pandemic in Italy. Their management team relied on accurate data to guide their surge response from the first recognition that the SARS-CoV-2 virus was in the community as COVID-19 patients began to appear. Their conventional management was to cohort patients based on their oxygen requirements and potential for aerosol (noninvasive ventilator treatment) as well as those placed on ventilators to maximize similar structure and space. As the ICU structure was filling up, they turned to the operative theater to increase their ventilator and monitoring space after the first days. While this required minimal investment of resources to reconfigure to limit exposure to staff and to have sufficient supplies, the team was identifying structures that could be adapted, repurposed with little creation to have space to manage the ventilator-dependent patients. The ward (structure) identified already had monitoring capability with the approach to create a filter zone between contaminated and noncontaminated spaces, increased warehouse (stuff), and sanitary (environmental services/housekeeping) space. By the 8th day, they had completed the transformation with cohort structures with space for oxygen-dependent patients. 


\section{TABLE 1}

\begin{tabular}{|lll|}
\hline \multicolumn{2}{|c|}{ Search Tool } & \\
Team Member & Concern & Mechanism \\
ED physician & Aerosol & Adapt \\
ED nurse & Droplet & Repurpose \\
Respiratory therapy & PPE: store/waste & Cohort \\
Radiology & PPE: don/doff & Create \\
Pharmacy & Traffic: patient & \\
Engineering & Traffic: staff & \\
IT & Traffic: radiology & \\
Housekeeping & Traffic: materials & \\
Logistic/warehouse & & \\
Registration & & \\
Nutrition & & \\
Security & & \\
Administration & & \\
\hline
\end{tabular}

This tool was used to screen literature for relevant content. ED, emergency department; IT, information technology; PPE, personal protective equipment; don, donning (putting); doff, doffing (removing).

\section{T3 = CREATION OF A CHECKLIST FOR COVID-19 SURGE STRUCTURE PLANNERS}

Through a review of prior MCI surge capacity literature, this study seeks to find guidance that can be used to understand the mechanisms ${ }^{44}$ to provide the structures necessary to meet the demands of the COVID-19 pandemic (Table 1). The creation of an expert team ${ }^{28}$ to provide critical infrastructure recommendations beyond the scope of the treatment team is imperative. Once the assembled team (Table 1) understands the likely timeline of the COVID-19 patient load demands on their hospital from conventional to contingency to crisis, they can grasp the challenges understanding the limitation of their structure to provide the necessary space to treat these patients while limiting exposure to staff while treating other patients and limiting their exposure.

This process begins with an agreement on how to create a single path into the ED. Saturation messaging in the community is crucial to prevent the ED from becoming a vector, overwhelmed with patients that do not require a detailed evaluation. The external tent structures now ubiquitous outside Italian EDs have become a fundamental screening space. This study does not address the creation or deployment of these or any other external structure or alternate care sites.

The team has to account for exposure by means of droplet from the patient's arrival in the parking lot. Patients should notify staff of their arrival while still in the car to obtain a surgical mask or to use another form of barrier over the face to limit droplets. Space for this staff can be created in or near the ED entrance. The team can do a walk-through as if they were the patient approaching the ED to understand where signage should be placed to direct the patient on a COVID-19 path in distinction from the "Clean" patient path, or all other patients. This can be done with poles and ropes if available or tape on the floor with arrows, especially if either the Clean or
FIGURE 3

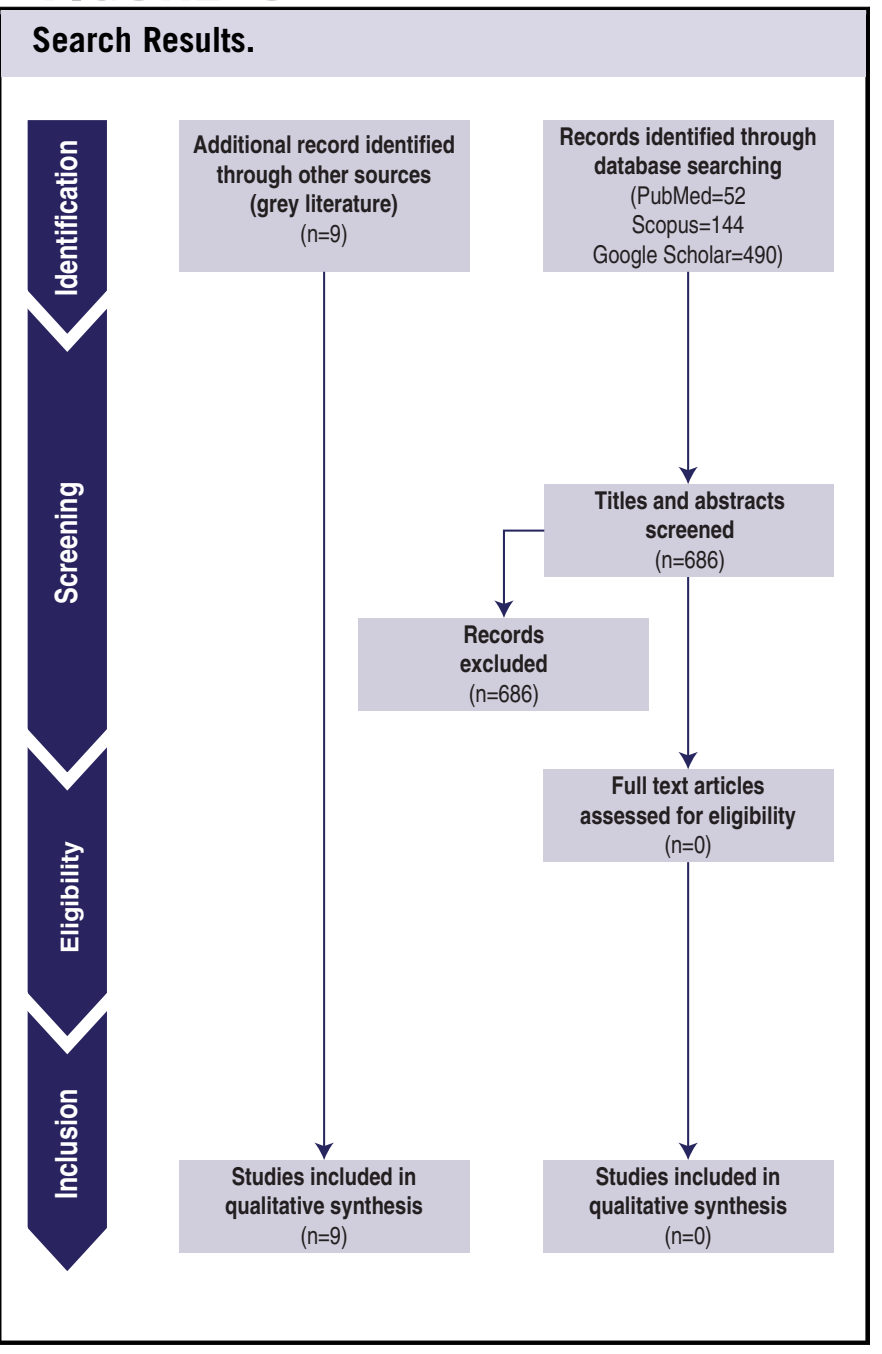

COVID-19 areas are not in the usual ED location in the hospital. Volunteers wearing masks or facial barriers that are more than 6 feet from either path can direct the patient to their treatment destination. Space for volunteers will require consideration if that is outside the entrance or within the structure of the ED to ensure they can manage their activities.

Typically, the ED waiting space is crowded with patients who are waiting to be assessed in triage, have been triaged and waiting to be examined, or waiting for results of tests or images ordered at triage. These patients are usually not sequestered or removed from patient family members. Now the team has to create a COVID-19 waiting space separate and distinct from the Clean waiting space. A distance of at least 6 feet from any COVID-19 family member should be ensured, because they may have been exposed and able to transmit the SARS-CoV-2 virus, and preferably not even close to any Clean patients or family members. The COVID-19 space should be well ventilated and in proximity to staff that can monitor those patients waiting to go to the ED treatment space. Some EDs feature vending machines and televisions 


\section{Scoping Search Results}

\section{Title}

Covid-19 Podcast From Italy

With Roberto Cosentini ${ }^{30}$

Source (Journal, Website...)

St. Emlyn's Blog

https://www.stemlynsblog.org/covid-19 podcast-from-italy-with-robertocosentini-st-emlyns/

EMplify Emergency Medicine Podcast

COVID-19 Update: An Interview With Andrea Duca, MD ${ }^{33}$

COVID-19: A Powerful Message From Italy ${ }^{34}$

COVID-19 Emergency

Department Response

Strategies ${ }^{35}$

Duty to Plan: Health Care, Crisis Standards of Care, and Novel Coronavirus SARS-CoV-2 ${ }^{36}$

National Strategic Plan for Emergency Department

Management of Outbreaks of https://www.acep.org/globalassets/sites/ COVID-1937

American College of Emergency Physicians

https://www.acep.org/globalassets/newdepartment-response-strategies.pdf

National Academy of Medicine

https://nam.edu/duty-to-plan-healthcare-crisis-standards-of-care-andnovel-coronavirus-sars-cov-2/

American College of Emergency Physicians acep/media/by-medical-focus/covid-19 https://blubrry.com/emplify/57487286/

episode-38-covid-19-update-aninterview-with-andrea-duca-md/

REBEL EM Blog

https://rebelem.com/covid-19-apowerful-message-from-italy/ pdfs/covid-19-for-emergencynational-strategic-plan_0320.pdf

\section{Team Member/Actor \\ Emergency department \\ Concer}

physicians

Timing for structural modifications

Treatment of COVID-19 patients

depending on oxygen and ventilation

Hospital medical direction Hospital admission increase

Emergency department Spread of the disease

physicians

\section{Timing to create dirty area}

Not enough space

PPE shortage

Shortage of equipment and supplies

Emergency department physicians

Spread of the disease

Change in respiratory diseases prevalence

Timing of changes

Hospital medical direction PPE shortage

Emergency medicine

\section{Oxygen shortage}

Risk of contamination/exposure

physicians

$$
\text { PPE shortage }
$$

Emergency medicine physicians

\section{Emergency medicine} physicians and hospital medical direction

\section{Mechanism (How To)}

Split ED into "Lung Diseases Unit" and "Other Diseases Unit"

Prepare in time - during the onset phase because presentation rate grow exponentially Divide the "Lung Diseases Unit" (contaminated path) into a high, medium, and low intensity areas

Create spaces

Create a contaminated/dirty Area and dedicated pathway

Progressively adapt to the increasing surge of patients

Create open space wards and waiting areas where patients are treated while waiting for hospital boarding

Cohort patients with COVID-19 to gain space Change gloves between patients

Rationalize use of equipment and materials Creation of 2 divided paths inside the ED: a contaminated and a clean one

Adapt and repurpose spaces to the increasing prevalence, remodeling on the base of oxygen sources and ventilators - dedicating most of the department to see acute respiratory patients. Leave a small clean area of the ED for non-suspect patients

Progressively change but expect an exponential increase of presentations so enact changes quickly.

Reduce shifts and dedicate personnel to the dirty path to reduce PPE wastage

Pay attention to oxygen administration Reduce episodes of donning and doffing/patient encounter

Increasing intercommunication and "door" exam Create a screening point outside of the hospital

Repurpose current rooms/areas to isolation rooms and infectious care areas

Cohorting patients

Boarding, discharge, and admission waiting areas. Separate areas for patients with respiratory symptoms

Maximize distance between patients

(at least 6 feet)

Protocols for people accompanying patients in waiting areas and visitors 


\section{Continued}

Source (Journal, Website...)

EUSEM Position Paper on

Emergency Medical System

Response to COVID-1938
European Society of Emergency Medicine position-paper-on-emergency-medicalsystems-response-to-covid-19
COVID-19 First Line - 10

Topics From the EDs

During Corona ${ }^{39}$

(Prima Linea COVID-19 - 10

cose... dai PS ai tempi del

Corona)

COVID-19 First Line Report -

Organizational Structure of

EDs Before and During the

Outbreak ${ }^{40}$

(Rapporto prima linea COVID

19 - Assetto organizzativo

gestionale dei PS/DEA

nell'ambito di focolaio

epidemico o pre-epidemico)

Checklist for Healthcare

Facilities: Strategies for

Optimizing the Supply of N95 https://www.cdc.gov/coronavirus/2019-

Respirators during the

COVID-19 Response ${ }^{41}$
Centers for Disease Control and Prevention

Italian Society of Emergency Medicine (Società Italiana Medicina

d'Emergenza-Urgenza)

https://www.simeu.it/w/articoli/

https:/www simeu it/w/articoli/

leggiArticolo/3964/leggi ncov/hcp/checklist-n95-strategy.html

\begin{tabular}{|c|c|}
\hline Team Member/Actor & Concern \\
\hline $\begin{array}{l}\text { Emergency medicine } \\
\text { physicians, hospital } \\
\text { medical direction, } \\
\text { cleaning service }\end{array}$ & Spread of disease \\
\hline Hospital medical direction & $\begin{array}{l}\text { Increased presentation and } \\
\text { admission rates }\end{array}$ \\
\hline Emergency department & Spread of the disease \\
\hline
\end{tabular}

Emergency department

Spread of the disease
Cleaning service

Emergency department

Emergency department

\section{PPE shortage}

Increased presentation rate

Spread of the disease

PPE shortage

Oxygen shortage

\section{Mechanism (How To)}

Protocols for decontamination

Opening unused areas,

patient cohorting, doubling up inpatient rooms Increase number of isolation rooms, possibly with negative pressure and adequate ventilation Favor physical barriers between patients

Specific triage areas for suspect patients Create patient dedicated waiting areas for suspects before boarding

Minimization of movements for suspect patients

( $E D$, radiology, bathrooms)

Cohorting of patients is reasonable

Maintain a distance of at least 1 meter

Don't allow visitors or co-operators in the area

Specific decontamination protocols

Treat waste from the contaminated area as a high risk biological material

Limit access to ED to patients with severe

symptoms. Asymptomatic or mild symptomatic patients should seek advice to GP

Increase stockpiles and supplies

Prepare to the surge at least 10 days before

Repurpose areas increasing possibility of ventilatory support

Reduce visits and activities to essentials Create "filter zones" (forward triage or screening areas)

Separate Areas for Suspect infectious patients Increase stockpiles and supplies increase stockpiles and supplies

Emergency Department Spread of the disease, PPE shortage
Use isolation rooms

Use physical barriers between patients

Maintain adequate ventilation 
to occupy those waiting, and the team will have to address the allocation and maintenance of these in relationship to the prior adapted, repurposed, or created triage waiting area structure or space. Registration of COVID-19 patients should be separate and distinct from Clean patients. This can be accomplished in the prior registration space or space that has been adapted, repurposed, or created to accommodate a computer desktop, a portable device on wheels, or a tablet for the registration personnel.

The depth and breadth of treating COVID-19 patients requires oxygen, compressed medical air, and vacuum for suction. ${ }^{45}$ Outlets and piping may be hidden behind walls created when space was repurposed to become an office, conference room, or other nontreatment areas. Ventilators and other machines may require different electrical outlets or local grids to avoid overloading any circuits. The treatment team members, physicians, and nurses are encouraged to discuss with the engineer team member the specific vital components of a reconfiguration.

Similarly, contaminated solid and fluid waste, as well as contaminated laundry and other trash, will require proper positioning in structures or spaces that were not designed for the sophisticated support required to treat the COVID-19 patients. The team's attention to this detail will provide the opportunity to maintain a secure infection control loop. Environmental services or housekeeping standard operating procedures during COVID-19 are essential with droplet control paramount to reduce exposure to all as well as rapid turnaround of space for the next patient. They will require pathways from their base of operations through the structure to their duty location with their advanced materials with the need to resupply at a location that may be closer than their base. Because these supplies may be of limited stock and valued by many, a secure location in the structure is required.

Central monitors may be able to be moved from 1 structure to another to create adapted repurposed or created space. This reconfigured space will have to accept computers or charging stations for portable electronic health record devices. The information technology and engineering team members may have innovative solutions to deploy these support assets with minimal cost and time to deploy, in safe and convenient locations.

The path that a patient takes from their ED space, with a door if available, unless in a cohort of similar patients, to radiology or their in-patient destination should be clearly marked as COVID-19 patients, with plastic or other barriers to prevent aerosol droplets landing on Clean hallways, walls, or doors along the way. The creation of filter zones, as mentioned by the team in Lodi using available construction plastic or plastic strips similar to the barriers in refrigeration compartments of stores that hang from the ceiling to the floor, provide a droplet barrier. Ideally, these can be obtained and stored before the need with anchor points along the locations in the structure.

Portable imaging machines are cumbersome and to be factored into the process of surge structure design. Also, beds, gurneys, and the associated IV poles, tables, and other materials that are required for a patient in the treatment space are to be accounted. One may best be served to take a gurney or bed with you to a structure that was not designed for in-patient treatment, such as a clinic or IV infusion center where there are only incline chairs or exam tables, to see if these patient support devices can maneuver in the tight spaces.

Most structures within a hospital have a just-in-time process to manage stuff to maintain organization, as a means to know what is used to restock and to charge the patient. In a pandemic, this will likely not be entirely possible to implement, although both the storage and resupply have to remain convenient, secure, and free from contamination unless approximate as necessary to maximize patient treatment. The pharmacy storage and distribution process will rapidly outpace any automated medication dispensing system (eg, BD Pyxis ${ }^{46}$ ). The team's reliance on the guidance of the pharmacy and nursing representatives will be necessary to assure an efficient loop for the treatment team to have what they need in the reconfigured structure.

\section{T4 = COLLECTION OF COMPLETED CHECKLISTS TO IMPROVE THE COVID-19 SURGE STRUCTURE PLANNING}

There is no argument that the evidence-based medicine approach using the Delphi method would have been in line with prior research in the field. ${ }^{27,28,36,38,39}$ These are extraordinary times, and the authors accept this limitation to produce a checklist to guide the team that has been tasked to reconfigure their structure for their surge COVID-19 response. The translational science intention is to create a checklist guide where none existed previously, fully aware that this checklist has not been vetted or verified or tested through simulation or live exercise. Translational science calls for feedback using the checklist (Table 3; an editable version is available as Online Supplemental Material 1) for the authors to continue this study to use to perform the T2 PRISMA review after a COVID-19 research body of work has been published on this topic. The checklist is to be used to collaborate with all relevant actors in real-time as the pandemic is unfolding to maximize the surge structure response in a timely manner, with minimal disruption to the non-COVID-19 patients in the facility, using available staff and stuff with the intent to return the structure to the prior state as soon as possible to resume the activities before this pandemic. 


\section{Surge Structure Checklists}

\section{TEAM MEMBER}

ED PHYSICIAN

ED NURSE

RESPIRATORY THERAPY

RADIOLOGY

PHARMACY

ENGINEERING

INFORMATION TECH. (IT)

HOUSEKEEPING

LOGISTIC/WAREHOUSE

REGISTRATION

NUTRITION

SECURITY

ADMINISTRATION

\section{STRUCTURE}

MECHANISM

Person in Charge Time to Change

Distance to ED

\begin{tabular}{llll}
\hline ADAPT & REPURPOSE COHORT
\end{tabular}

\section{CONCERN}

Person in Charge

ONGOING

ADDRESSED

N/A

TASKS/NOTES

AEROSOL (DOOR?)

DROPLET (filter zone)

PPE: STORE/WASTE

PPE: DON/DOFF

TRAFFIC: PATIENT

TRAFFIC: STAFF

TRAFFIC: RADIOLOGY

TRAFFIC: MATERIALS

BED/STRETCHER/CHAIR

POLES

TABLE

ELECTRIC

GASES

WATER/TRASH

VENT/NIV

\section{About the Authors}

CRIMEDIM - Research Center in Emergency and Disaster Medicine, Università del Piemonte Orientale, Novara, Italy.

Correspondence and reprint requests to Matteo Paganini, Research Center in Emergency and Disaster Medicine (CRIMEDIM). Università del Piemonte Orientale. Via Lanino 1, 28100 Novara, Italy (e-mail: matteo.paganini@ uniupo.it)

\section{Supplementary material}

To view supplementary material for this article, please visit https://doi.org/10.1017/dmp.2020.57

\section{REFERENCES}

1. Sustainable Development Goals. United Nations. https://www.un.org/ sustainabledevelopment/health/. Accessed March 21, 2020.
2. The determinants of health expenditure. A country-level panel data analysis. World Health Organization. https://www.who.int/health_financing/ documents/cov-report_e_11-deter-he/en/. Published 2011. Accessed March 20, 2020.

3. Bernard C. Lecons Sur Les Phenomenes de La Vie Communs Aux Animaux and Aux Vegetaux. Paris: Bailliere; 1878.

4. Modell H, Cliff W, Michael J, et al. A physiologist's view of homeostasis. Adv Physiol Educ. 2015;39(4):259-266. doi: 10.1152/advan.00107. 2015

5. Coronavirus Disease 2019 (COVID-19) Situation Report - 49. World Health Organization. https:/www.who.int/emergencies/diseases/novelcoronavirus-2019/situation-reports/. Accessed March 20, 2020.

6. Coronavirus Disease 2019 (COVID-19) Italian Situation, Press release. Italian Civil Protection. http://www.protezionecivile.it/web/guest/mediacommunication/press-release/detail/-/asset_publisher/default/content/ coronavirus-sono-7-985-i-positivi. Accessed March 20, 2020.

7. Strauss-Riggs K, Yeskey K, Miller A, et al. Translating battlefield practices to disaster health. Disaster Med Public Health Prep. 2017;11(4):510-511. doi: $10.1017 / \mathrm{dmp} .2016 .196$ 
8. What is Translational Science? Tufts Clinical Science and Translational Institute. https://www.tuftsctsi.org/about-us/what-is-translational-science/. Accessed March 19, 2020.

9. Translational Science Spectrum 2015. National Center for Advancing Translational Sciences. https://ncats.nih.gov/files/translation-factsheet. pdf. Accessed March 19, 2020.

10. Turner M, Landford T. Grey literature in health. University of Canberra. https://canberra.libguides.com/greyliterature. Accessed March 20, 2020.

11. Kelen GD, McCarthy ML. The science of surge. Acad Emerg Med. 2006;13(11):1089-1094. doi: 10.1197/j.aem.2006.07.016

12. Barbisch DF, Koenig KL. Understanding surge capacity: essential elements. Acad Emerg Med. 2006;13(11):1098-1102. doi: 10.1197/j.aem. 2006.06.041

13. Brown ED, Williams BK. Resilience and resource management. Environ Manage. 2015;56(6):1416-1427. doi: 10.1007/s00267-015-0582-1

14. Adger WN. Social and ecological resilience: are they related? Prog Hum Geogr. 2000;24(3):347-364. doi: 10.1191/0309132007 01540465

15. Ziglio E, Azzopardi-Muscat N, Briguglio L. Resilience and 21st century public health. Eur J Public Health. 2017;27(5):789-790. doi: 10.1093/ eurpub/ckx116

16. Cristian B. Hospital resilience: a recent concept in disaster preparedness. J Crit Care Med. 2018;4(3):81-82. doi: 10.2478/jccm2018-0016

17. Hogan DE, Waeckerle JF, Dire DJ, et al. Emergency department impact of the Oklahoma City Terrorist Bombing. Ann Emerg Med. 1999;34(2):160-167. doi: 10.1016/s0196-0644(99)70224-6

18. Kaji A, Koenig KL, Bey T. Surge capacity for healthcare systems: a conceptual framework. Acad Emerg Med. 2006;13(11):1157-1159. doi: 10.1197/j.aem.2006.06.032

19. Nozaki H, Hori S, Shinozawa Y, et al. Secondary exposure of medical staff to sarin vapor in the emergency room. Intensive Care Med. 1995;21(12):1032-1035. doi: 10.1007/bf01700667

20. Mothershead JL, Tonat K, Koenig KL. Bioterrorism preparedness III: state and federal programs and response. Emerg Med Clin North Am. 2002;20(2):477-500. doi: 10.1016/s0733-8627(02)00004-4

21. Severe Acute Respiratory Syndrome (SARS). Centers for Disease Control and Prevention. https://www.cdc.gov/sars/index.html. Accessed March 21, 2020.

22. Smith EC, Burkle FM, Holman PF, et al. Lessons from the front lines: the prehospital experience of the 2009 Novel H1N1 outbreak in Victoria, Australia. Disaster Med Public Health Prep. 2009;3(S2):S154-S159. doi: 10.1097/dmp.0b013e3181 be 8250

23. Danielsson N, ECDC Internal Response Team, Catchpole M. Novel coronavirus associated with severe respiratory disease: case definition and public health measures. Eurosurveillance. 2012;17(39):pii 20282. doi: 10.2807/ese.17.39.20282-en

24. Shoman H, Karafillakis E, Rawaf S. The link between the West African Ebola outbreak and health systems in Guinea, Liberia and Sierra Leone: a systematic review. Glob Health. 2017;13(1):1. doi: 10.1186/s12992-0160224-2

25. Hick JL, Barbera JA, Kelen GD. Refining surge capacity: conventional, contingency, and crisis capacity. Disaster Med Public Health Prep. 2009;3(S1):S59-S67. doi: 10.1097/dmp.0b013e31819f1ae2

26. Li R, Pei S, Chen B, et al. Substantial undocumented infection facilitates the rapid dissemination of novel coronavirus (SARS-CoV2). Science. 2020:eabb3221. doi: 10.1126/science.abb3221

27. Bradt DA, Aitken P, FitzGerald G, et al. Emergency department surge capacity: recommendations of the Australasian Surge Strategy Working Group. Acad Emerg Med. 2009;16(12):1350-1358. doi: 10.1111/j.1553. 2712.2009.00501.x
28. Hick JL, Christian MD, Sprung CL. Chapter 2. Surge capacity and infrastructure considerations for mass critical care. Intensive Care Med. 2010;36(S1):11-20. doi: 10.1007/s00134-010-1761-4

29. Riou J, Althaus CL. Pattern of early human-to-human transmission of Wuhan 2019 novel coronavirus (2019-nCoV), December 2019 to January 2020. Euro Surveill. 2020;25(4). doi: 10.2807/1560-7917.es. 2020.25.4.2000058

30. Covid-19 Podcast from Italy with Roberto Cosentini. St Emlyn's Blog. https://www.stemlynsblog.org/covid-19-podcast-from-italy-with-robertocosentini-st-emlyns. Accessed March 21, 2020.

31. Tricco AC, Lillie E, Zarin W, et al. PRISMA extension for scoping reviews (PRISMA-ScR): checklist and explanation. Ann Intern Med. 2018;169(7):467. doi: 10.7326/m18-0850

32. PubMed. National Library of Medicine, National Center for Biotechnology Information. https://www.ncbi.nlm.nih.gov/pubmed/. Accessed March 21, 2020.

33. COVID-19 update: an interview with Andrea Duca, MD. EMplify emergency medicine podcast. https://blubrry.com/emplify/57487286/episode38-covid-19-update-an-interview-with-andrea-duca-md/. Accessed March 21, 2020.

34. COVID-19: a powerful message from Italy. REBEL EM Blog. https:// rebelem.com/covid-19-a-powerful-message-from-italy/. Accessed March 21, 2020 .

35. COVID-19 Emergency Department Response Strategies. American College of Emergency Physicians. https:/www.acep.org/globalassets/ new-pdfs/covid-19-for-emergency-department-response-strategies.pdf. Accessed March 20, 2020.

36. National Academy of Medicine. Duty to plan: health care, crisis standards of care, and novel coronavirus SARS-CoV-2. https://nam.edu/duty-toplan-health-care-crisis-standards-of-care-and-novel-coronavirus-sars-cov-2/. Accessed March 20, 2020.

37. National Strategic Plan for Emergency Department Management of Outbreaks Of COVID-19. American College of Emergency Physicians. https://www.acep.org/globalassets/sites/acep/media/by-medical-focus/ covid-19-national-strategic-plan_0320.pdf. Accessed March 21, 2020.

38. EUSEM Position paper on Emergency Medical Systems response to COVID-19. European Society of Emergency Medicine. https://eusem. org/news/505-eusem-position-paper-on-emergency-medical-systemsresponse-to-covid-19. Accessed March 21, 2020.

39. COVID-19 First Line - 10 topics from the EDs during Corona. Italian Society of Emergency Medicine. https://www.simeu.it/w/articoli/leggi Articolo/3964/leggi. Accessed March 20, 2020.

40. COVID-19 First Line Report - organizational structure of EDs before and during the outbreak. Italian Society of Emergency Medicine. https://www. simeu.it/w/articoli/leggiArticolo/3992/leggi. Accessed March 21, 2020.

41. Checklist for Healthcare Facilities: Strategies for optimizing the supply of N95 respirators during the COVID-19 response. Centers for Disease Control and Prevention. https:/www.cdc.gov/coronavirus/2019-ncov/ hcp/checklist-n95-strategy.html. Accessed March 20, 2020.

42. Faccincani R, Lennquist S. How to surge to face SARS-CoV-2 outbreak. Disaster Med Public Health Prep. In press.

43. Gagliano A, Villani P, Co F, et al. 2019-ncov's epidemic in middle province of northern Italy: impact, logistic \& strategy in the first line hospital. Disaster Med Public Health Prep. 2020:1-15.

44. Hick JL, Einav S, Hanfling D, et al. Surge capacity principles. Chest. 2014;146(4):e1S-e16S. doi: 10.1378/chest.14-0733

45. Einav S, Hick JL, Hanfling D, et al. Surge capacity logistics. Chest. 2014;146(4):e17S-e43S. doi: 10.1378/chest.14-0734

46. BD Pyxis MedStation ES. BD. https://www.bd.com/en-us/offerings/ capabilities/medication-and-supply-management/medication-and-supplymanagement-technologies/pyxis-medication-technologies/pyxis-medstationes-system. Accessed March 20, 2020. 